\title{
Development of optical MEMS-based micromirror arrays on flexible substrate for curvilinear surfaces
}

\author{
Muhammad Rizwan Amirzada ${ }^{1,2}$ (D) Qingdang $\mathrm{Li}^{1,3} \cdot$ Hartmut Hillmer $^{1}$
}

Received: 16 March 2020 / Accepted: 27 March 2021 / Published online: 18 April 2021

(c) The Author(s) 2021

\begin{abstract}
This research work is targeting to demonstrate that micromirror arrays can also be fabricated successfully on flexible substrates - not only on glass substrates. The paper reports on the technological data of a successful process on a flexible substrate for curvilinear surfaces. Polyethylene naphthalate substrate was selected for its low cost, strong resistance against chemicals which are used in fabrication process and sufficient optical transmission for the visible and near infrared spectrum. Finally, electrostatic actuation of the fabricated micromirror structures is proven experimentally.
\end{abstract}

Keywords Optical MEMS $\cdot$ Micromirros $\cdot$ Flexible substrate $\cdot$ Polyethylene naphthalate

\section{Introduction}

Conventional electronic circuits are fabricated on planar hierarchically nano-patterned semiconductor substrates. However, the substrates reveal mechanical rigidity and stiffness which limit the degree of utility due to its (1) inability to allow also soft curvilinear morphology, (2) brittle nature and (3) limited optical transparency of the substrate allowing only wavelengths above the respective band gap wavelengths. However, flexible substrates combined with the polymeric framework would allow light-weighted thin-film architectures, wearability, reduced restrictions in optical transparency, and morphological configuration to curvilinearity (Lu and Kim 2013; Yaojian and Kang 2016; Malik and Kandasubramanian 2018).

Advances in various organic semiconductor technologies on flexible substrates have led to an immense production of devices for applications such as wearable health monitoring

Muhammad Rizwan Amirzada

mamirzada@numl.edu.pk

1 Institute of Nanostructure Technologies and Analytics (INA), Center for Interdisciplinary

Nanostructure Science and Technology (CINSaT), Universität Kassel, Heinrich-Plett-Str. 40,

34132 Kassel, Germany

2 Faculty of Engineering and Computer Sciences, National University of Modern Languages, Islamabad, Pakistan

3 Qingdao University of Science and Technology, Sino German Technical University, 575\#, No. 99 Songling Road, Qingdao 266061, People's Republic of China 
systems, photovoltaic cells, and portable consumer electronics (Zhu et al. 2016; Wang et al. 2017; Liu et al. 2018). These flexible gadgets have a long history and analysis to create diverse hardware on an adaptable substrate. Since the substrate is the main element for stretchability and flexibility of diverse flexible hardware, allowing for long-term mechanical reliability and comfort level. The materials like silicone, rubbers, polymers and other organic materials have great potential as substrate. Flexible devices have an abundant tale and critics, and required much effort to create diverse hardware on a flexible substrates, such as Photovoltaic (PV) cells, Liquid Crystal Displays (LCDs), Thin Film Transistor (TFT), Micro Electro Mechanical Systems (MEMS) and so on (Cheng et al. 2016; Ge and Cretu 2019). Interest for adaptable devices raised during the 1960s, when the solar cell arrays were fabricated on an adaptable substrate (Daniel 1960). Essentially, a plastic flexible substrate is the increasingly basic substrate for adaptable gadgets, for example, thinfilm transistor (TFTs), solar cells, flexible batteries and LEDs and so on as appearing in Fig. 1.

One important application of MEMS is Micromirror Arrays (MMA) which are mirror structures shortened to several up to hundreds of micrometer $(\mu \mathrm{m})$. MEMS-based MMA for smart windows can be used for active daylight steering, glare protection and climatization inside the building (Hillmer et al. 2018; Iskhandar et al. 2019). To overcome limitations in current lighting, heating and air-conditioning in state-of-the-art buildings intelligent glazings using MEMS-based MMA have been developed on glass substrates(Hillmer et al. 2018; Iskhandar et al. 2019).

Figure 2 depicts a schematic of a single mirror out of the whole array. Initially, the micromirror is open and can be closed by biasing the top and bottom electrodes via applying an external actuation voltage across the electrodes typically in the range of 12-60 V. The micromirrors measure e.g. $150 \times 400 \mu \mathrm{m}^{2}$ (Hillmer et al. 2018).

The light steering concept using MMAs in smart glazings is described in detail in Refs. (Hillmer et al. 2018; Iskhandar et al. 2019).

The main aim of this research work is to fabricate these MMA for the first time on flexible substrates, as these substrates are thin, lightweight and bendable and can be

Fig. 1 Some examples of flexible electronics a flexible TFT structures (Shih and Chin 2017) b flexible solar cells (Yang et al. 2019) c flexible electronic battery (Koo et al. 2012), d flexible random access memory (RAM) (Gan et al. 2019)
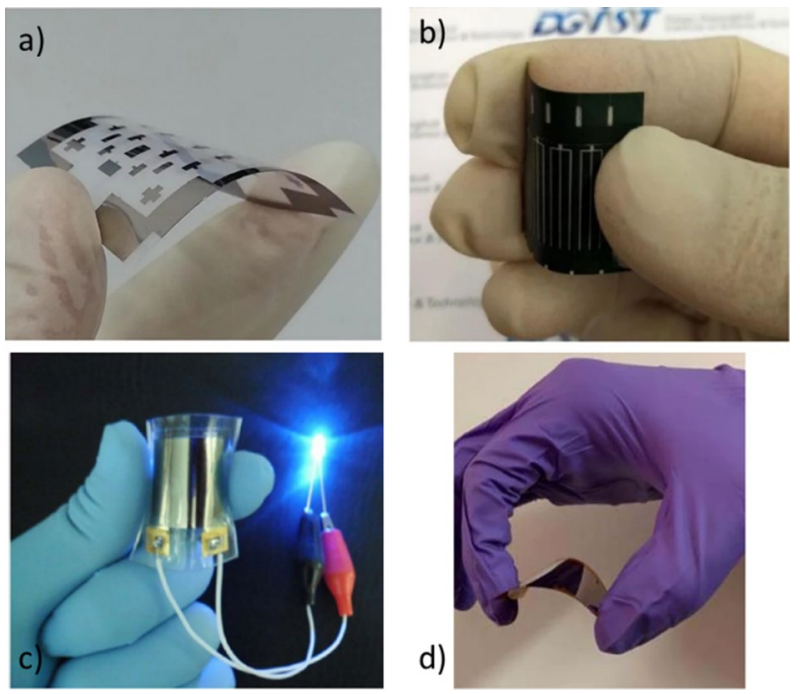

d)

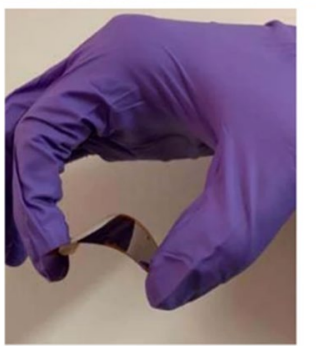




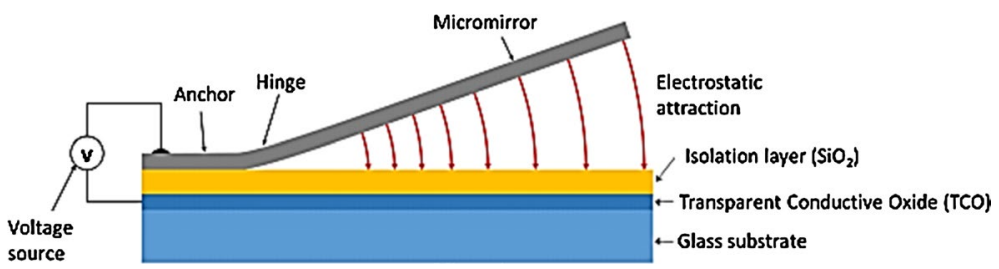

Fig. 2 Schematic of a single mirror out of the array. The single micromirror structure comprises of micromirror layer which is fixed at one end as top electrode (Anchor area) insulated from the bottom electrode (here: transparent conductive oxide). The two conductive electrodes are isolated from each other by silicon dioxide $\left(\mathrm{SiO}_{2}\right)$. Original figure from Iskhandar et al. (2019)

implemented on a curved area surface as compared to a flat surface (Yao et al. 2019; Park et al. 2019). The main goal is also to present a working process recipe and to demonstrate the operation and actuation of the MMA, finally. These lightweight and bendable micromirror substrates can be implemented e.g. on the front and back mirror of vehicles, photovoltaic concentrators or any curved surface. Note that bending of float glass is often used today in cars or buildings, but after shaping it stays inflexible and rigid. Thus, the main advantage of flexible substrates for MMA is the possibility to actuate the bending shape actively or to fit it into already existing bent shapes.

There are three substrates which can be classified as flexible substrates, thin glass whose thickness is several hundred micrometers, metal foils which are very thin and can be bend at a certain angle and transparent polymer foils (Wong and Salleo 2009; Zhou et al. 2018). The first two types of substrates are not suitable as metal foils are not transparent and the glass substrate is not flexible enough and brittle to meet the purpose so the only choice left is to use a transparent polymer foil (Wong and Salleo 2009).

\section{Experimental work}

\subsection{Suitable substrate selection}

The first step of the transformation of the MMA to a flexible substrate is to select an appropriate substrate which has adequate properties i.e. (1) it can tolerate the uppermost temperatures occurring during the fabrication process, (2) it has sufficient optical properties in terms of light transmission, and (3) it must show resistance against certain chemicals which are used during the fabrication process. After extensive screening the market, the Polyethylene Naphthalate (PEN) flexible substrate was selected for the fabrication process. The datasheet of the substrate shows that PEN substrate can sustain the temperature of $120{ }^{\circ} \mathrm{C}$ which is the glass transition temperature for PEN and hence makes it suitable for MMA fabrication process. The above-mentioned PEN substrate was provided by Dupont Teijin Films (Teonex® Q65F).

\subsubsection{Optical properties}

After the suitable selection of the flexible substrate, the optical characteristics of the PEN substrate were tested. This optical test is very important as the MMA are intended to be used in smart bent optical surfaces or glazing, requiring sufficient substrate transparency. 
Experimental spectral transmission studies revealed a weak variation on wavelength as shown in Fig. 3. In average approximately $80 \%$ of the light is transmitting through the PEN substrate and approximately $70 \%$ average light transmission is measured after Indium Tin Oxide (ITO) layer deposition.

\subsubsection{Chemical stability test}

After optical tests, chemical stability tests were carried out on a PEN substrate. The chemicals which intended to be used during the fabrication of MMA were tested against the PEN substrate. The small pieces of PEN substrate were cut and immersed in acetone and isopropyl alcohol for the duration of one hour. The second test was carried out with potassium hydroxide $(\mathrm{KOH})$ and small pieces of PEN substrate were immersed in $\mathrm{KOH}$ for $10 \mathrm{~min}$. Finally, the PEN substrate was interacting with an aluminum etchant which is a mixture of $\mathrm{HNO}_{3}, \mathrm{H}_{3} \mathrm{PO}_{4}, \mathrm{CH}_{3} \mathrm{COOH}$ and $\mathrm{H}_{2} \mathrm{O}$. It was found that the PEN substrate was not affected by all the above-mentioned chemicals and hence make the selected substrate suitable for MMA fabrication.

\subsubsection{Thermal test}

During the fabrication of the MMA process, the maximum temperature which is commonly used in the Plasma Enhanced Chemical Vapor Deposition (PECVD) procedure is $300{ }^{\circ} \mathrm{C}$. As per datasheet of Teonex $Q 65 F$ substrate, the melting point of the PEN substrate is $269{ }^{\circ} \mathrm{C}$. So, it was not possible to use the PEN substrate e.g. at $300{ }^{\circ} \mathrm{C}$. In our PECVD system (used for deposition of $\mathrm{SiO}_{2}$ layer), the substrate holder temperature can be set to values between $60{ }^{\circ} \mathrm{C}$ and $300{ }^{\circ} \mathrm{C}$, but the quality of the deposited layer is found to decrease, as lower the substrate temperature is (Amirzada et al. 2016). For the thermal test, PEN substrate was again cut into reasonable sizes which were heated on a hotplate from 80 to $240{ }^{\circ} \mathrm{C}$ with an increment of $40{ }^{\circ} \mathrm{C}$ for 15 min each. The PEN substrates have shown good resistance against the temperature from 80 to $200{ }^{\circ} \mathrm{C}$ without deformation. However, when the substrate was heated above $240{ }^{\circ} \mathrm{C}$ the PEN substrate changed its shape, considerably and irreversibly.

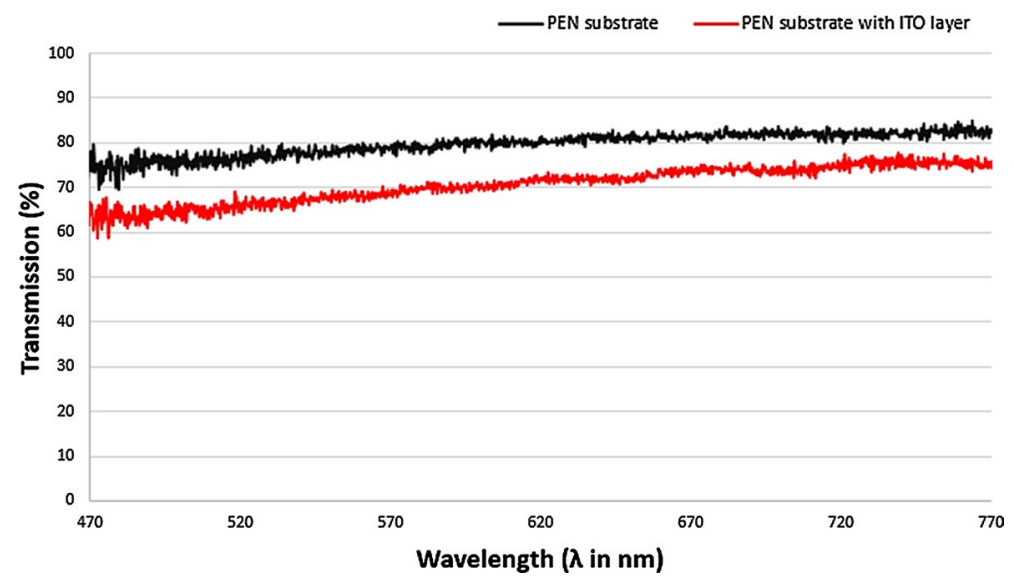

Fig. 3 Spectral transmission of a PEN foil of $125 \mu \mathrm{m}$ thickness with and without ITO layer 


\subsection{Fabrication process on flexible substrate}

Compared to the technological on glass substrates the technological process is different on PEN substrates. After the selection of a suitable substrate, the process of fabrication has been started. The first step of the fabrication is to deposit a transparent conducting layer on PEN substrate which will act as bottom electrode for MMA.

\subsubsection{Deposition of bottom electrode}

For the deposition of the bottom electrode, the deposited material must be conductive and transparent (transparent optical conductors, TOC). Using Ion beam sputter deposition, $100 \mathrm{~nm}$ thick layer of Indium Tin Oxide (ITO) was deposited on the PEN substrate whose dimensions are $10 \times 10 \mathrm{~cm}^{2}$ having a specific electrical resistivity of $10^{18} \Omega \mathrm{cm}$ according to the datasheet (the electrical conductivity is $10^{-16} \mathrm{~S} / \mathrm{m}$ ). After the deposition of the ITO bottom electrode, the conductivity and transmission of the ITO bottom electrode were measured. The measured ITO electrical conductivity on PEN substrates is $(1.02 \pm 0.04) \times 10^{6} \mathrm{~S} / \mathrm{m}$. The transmission is slightly increasing with rising wavelength as shown in Fig. 3. The corresponding deposition parameters are shown in the Table 1.

\subsubsection{Insulation layer deposition}

After the deposition of the ITO bottom electrode, $\mathrm{SiO}_{2}$ was deposited by PECVD as an insulation layer. The melting point and glass transition temperatures of the PEN substrates are $269^{\circ} \mathrm{C}$ and $121{ }^{\circ} \mathrm{C}$, respectively, according to the datasheet. This restricts the PECVD process temperature to $120^{\circ} \mathrm{C}$ or below.

For better insulation, a new approach was used in addition, where a stack of five layers was deposited one after another at different temperatures rather than only one layer. The main advantage of this technique is to reduce the dielectric breakdown effect in MMA structures (Wojtyra and Lesyk 2017). Each layer has a thickness of $200 \mathrm{~nm}$ and range of temperature was varied from 70 to $110^{\circ} \mathrm{C}$ with an increment of $10^{\circ} \mathrm{C}$. The PECVD parameters for the insulation layer deposition are shown in the following Table 2.

\subsubsection{Photolithography for sacrificial layer structuring}

After the insulation layer deposition, the next step in the fabrication of MMA is to structure the sacrificial layer using photolithography (Fig. 4a). Since a PEN substrate is flexible, lightweight and very thin as compared to a glass substrate, some modifications were required in the photolithography process. Before applying photoresist on

Table 1 Parameter details for deposition of the indium tin oxide (ITO)

\begin{tabular}{lll}
\hline Process parameters & First ion source & Second ion source \\
\hline Gas stream & Xe 03 sccm & $\mathrm{O}_{2} 09 \mathrm{sccm}$ \\
Power & $240 \mathrm{~W}$ & - \\
Beam & $1000 \mathrm{~V}(65 \mathrm{~mA})$ & - \\
Supply voltage (current) & $100 \mathrm{~V}(2.5 \mathrm{~mA})$ & - \\
Pulse & $1 \mathrm{kHz}(40 \%)$ & - \\
\hline
\end{tabular}


Table 2 Parameter details for silicon dioxide deposition using PECVD

\begin{tabular}{ll}
\hline Process characteristics elements & Value \\
\hline $2 \% \mathrm{SiH}_{4}-\mathrm{N}_{2}$ flow (sccm) & 430 \\
$\mathrm{~N}_{2} \mathrm{O}$ flow $(\mathrm{sccm})$ & 710 \\
$\mathrm{HF}$ power $(\mathrm{W})$ & 20 \\
$\mathrm{LF}$ power $(\mathrm{W})$ & 20 \\
Pressure (Torr) & 1 \\
Temperature $\left({ }^{\circ} \mathrm{C}\right)$ & $70-110^{\circ} \mathrm{C}$ \\
\hline
\end{tabular}
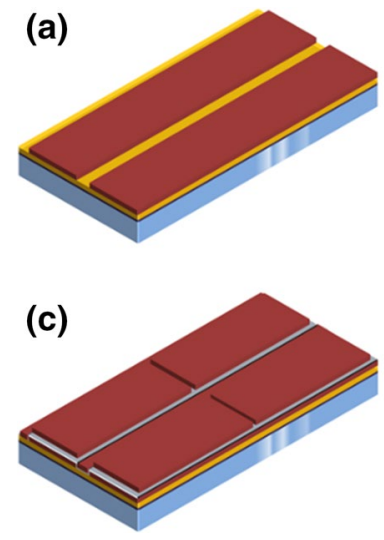
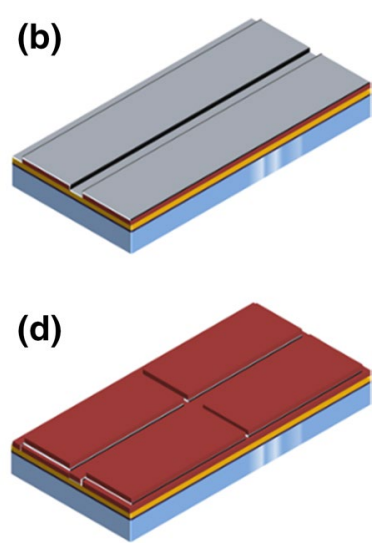

Fig. 4 a Schematic diagram of a sample with insulation layer (in yellow) and sacrificial layer (in maroon) before the aluminum deposition b sample after the aluminum deposition c sample after second photolithography but before aluminum etching $\mathbf{d}$ sample after aluminum etching. (Color figure online)

PEN substrates, the substrates were heated to $110{ }^{\circ} \mathrm{C}$ to remove the moisture and then an adhesion promotor (Ti Prime) was applied by spin coating (6000 rpm, $40 \mathrm{~s}$ ). Next, positive photoresist AZ 1505 was applied on the processed PEN substrate using the same spin coating process. The substrate was soft baked at $90{ }^{\circ} \mathrm{C}$ for $5 \mathrm{~min}$. The next step in the photolithography process is the exposure of the photoresist by ultraviolet (UV) light.

As PEN substrate is very thin and lightweight as compared to a glass substrate, has different refractive index and different UV reflection properties there are several changes required and some challenges in the photolithography process. The main challenge in the exposure process is to have a uniform exposure of UV light all over the surface so that whole patterns can be transferred from the mask to the substrates. The PEN substrate is not regularly flat and is not hard as compare to glass. To get uniformity in exposure over all the surface, the hard contact mode was used during the exposure of UV light instead of soft contact mode in which a small gap between mask and substrate exists. By using the hard contact mode during the UV exposure, the photomask applies a little pressure on the PEN substrate to eliminate the curvy nature to obtain a uniform exposure all over the surface. As a result of the used hard contact mode exposure, the quality of the photolithography process is more or less identical to the soft contact mode used for processing MMAs on glass substrates. Furthermore, the exposure and development time was also modified because of the nature of the substrate and after thorough research, the exposure time of UV light exposure was optimized to $4.2 \mathrm{~s}$ and $\mathrm{KOH}$ development time was optimized to $10 \mathrm{~s}$. 


\subsubsection{Mirror layer deposition and mirror structuring}

After the structuring the resist sacrificial layer via photolithography, aluminum mirror layers were deposited. The layers are uniform in lateral direction, i.e. the expand over the whole sample (Fig. 4b). Two separate layers of aluminum were deposited in the vertical direction using physical vapor deposition (PVD). The key factor for maintaining the shape of the mirror is the layer thicknesses of the deposited aluminum. Several samples have been deposited by different layer combinations and after a thorough investigation related to stress produced inside the layers, it was found that the best combination for a double mirror layer was $225 \mathrm{~nm} / 225 \mathrm{~nm}$ at a vacuum level of $3.5 \times 10^{-6} \mathrm{mbar}$ for our flexible substrates. Mirror structuring was performed via another photolithography process using the same parameters but different photomask (Fig. 4c). After development, the photoresist covers only the desired aluminum portion to be protected and prevents it etching. In the area which are not covered by the photoresist, the aluminum was removed using an aluminum etchant (mixture of $\mathrm{H}_{3} \mathrm{PO}_{4} 73 \%, \mathrm{HNO}_{3} 3.1 \%, \mathrm{CH}_{3} \mathrm{COOH}$ $3.3 \%$ and $\mathrm{H}_{2} \mathrm{O} 20.6 \%$ ) as shown in Fig. 4d).

In the final step, the sacrificial resist layer which was deposited during the first lithography process, is now removed by acetone. After removing the sacrificial layer, the mirror structures were released due to internal stress of aluminum layers which can be seen in Fig. 5. Four different aluminum layer combinations (in red below the corresponding four SEMs in Fig. 5) have been deposited by PVD on four different samples. All four samples have been finally processed to MMAs and characterized by SEM.

Our first main goal of this study, to demonstrate the feasibility of MMA fabrication on PEN substrates is met.

\section{Electrostatic actuation of micromirror arrays}

After the successful fabrication of MMAs on PEN substrates, the second main goal to demonstrate proper electrostatic actuation was envisaged.

Fig. 5 SEM images of micromirrors including 4 different aluminum layer combinations, respectively, deposited on 4 different samples by PVD

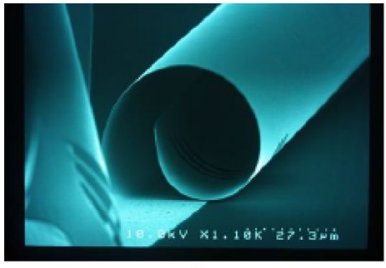

$150 \mathrm{~nm} / 150 \mathrm{~nm}$

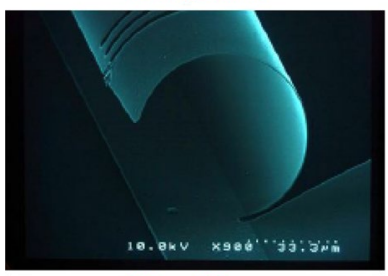

$225 \mathrm{~nm} / 225 \mathrm{~nm}$

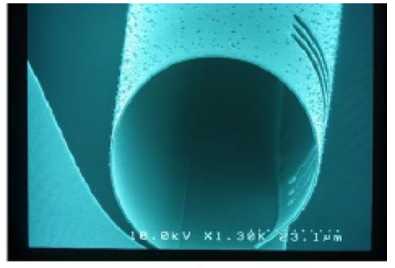

$190 \mathrm{~nm} / 190 \mathrm{~nm}$

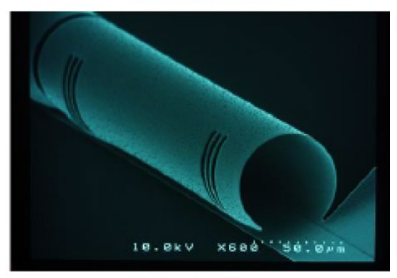

$240 \mathrm{~nm} / 290 \mathrm{~nm}$ 
1. $90 \mathrm{~V} \mathrm{DC}$ were found to be required for full closing on PEN substrates. This is in the range of the actuation voltages between 80 and $100 \mathrm{~V}$, which was typical for our technology start developing MMAs on glass substrates. After steady improvements in the process the actuation voltages typically range between 12 and $60 \mathrm{~V}$ today for our MMAs on glass substrates (Hillmer et al. 2018). We expect decreasing actuation voltages also for MMAs on PEN substrates after further optimization. However, we expect that the actuation voltages will not be as low as $12 \mathrm{~V}$ at the end for PEN substrates, for identical lateral geometries, due to the following reason: the deposition temperature of the insulation layer has to be lower (from 70 to $110^{\circ} \mathrm{C}$ ) for PEN substrates as compared to a glass substrate (up to $300{ }^{\circ} \mathrm{C}$ ).

2. For the MMAs on PEN substrates minimum and maximum transmittance of $1 \%$ and $50 \%$ has been measured. For MMAs on glass substrates we measured minimum and maximum transmittance of $0.01 \%$ and $65 \%$, respectively Wojtyra and Lesyk (2017). However, we expect that the values obtained on glass substrates will not be fully achievable on PEN substrates. Due to the following 2 reasons: (a) $\mathrm{SiO}_{2}$ layers on PEN substrates reveal higher roughness due to lower deposition temperature. Note that PECVD substrate temperature determines the surface quality and refractive index of the deposited layer (Amirzada et al. 2016). (b) In addition, the optical transmittance of the PEN substrates (approx. 80\%) is already lower than that of glass substrates (>90\%).

MMA samples in, both, open and closed state in bent substrate condition are presented in Fig. 6 in interaction with an external light source. The dynamic electrostatic actuation is visualized in a movie which can be found via the following link https://t1p.de/PEN-Micromirror.

\section{Conclusion}

In this work, our micromirror array design was successfully fabricated for the first time on flexible PEN substrates. PEN substrates were chosen because of their low cost, good transparency, and other benefits. Before starting the fabrication process, PEN substrates first had to pass severe qualification examinations: (1) tolerance of temperatures occurring during the fabrication process, (2) enough optical light transmission and (3) resistance against all chemicals used during the fabrication process. The mirror consisting of a double aluminum layer deposited using the PVD were found to be optimum for the PEN substrate process for $225 \mathrm{~nm} / 225 \mathrm{~nm}$, each. After fabrication electrostatic actuation was demonstrated and a totally open position was measured for $90 \mathrm{~V}$. In addition, a minimum and maximum transmittance of $1 \%$ and $50 \%$ was measured. 
Fig. 6 Different states of micromirrors on a flexible substrate $\mathbf{a}$ open state in bent position $\mathbf{b}$ closed state in bent position
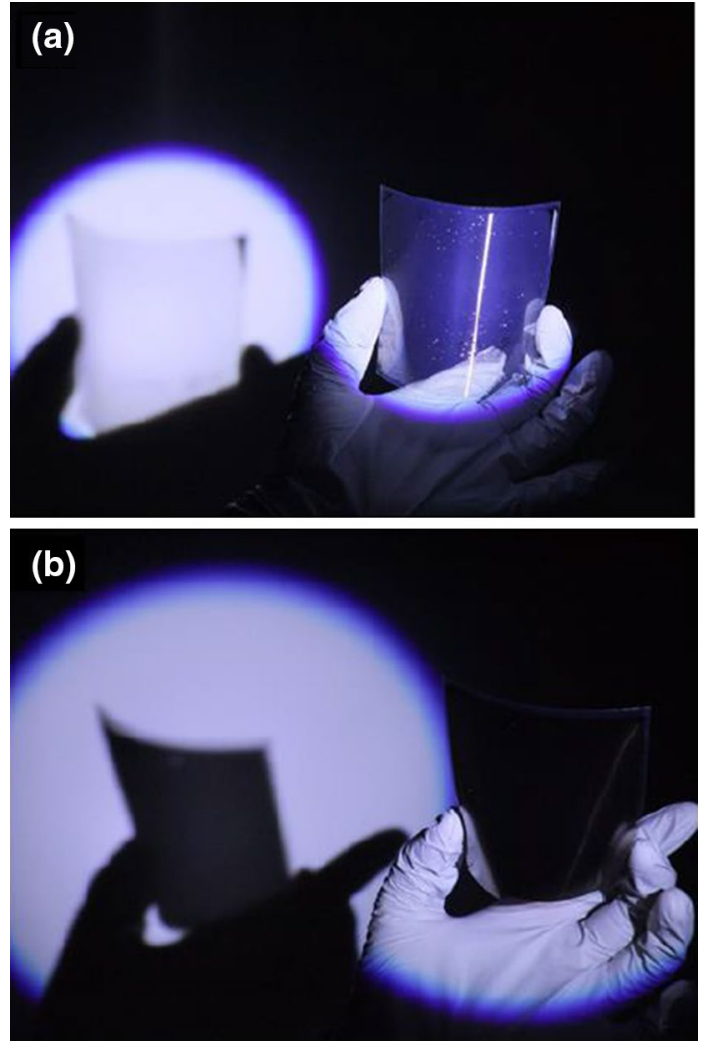

Acknowledgements The authors thank V. Viereck, A. Tatzel and all the colleagues in Institute of Nanostructure Technology and Analytics for fruitful discussions and technological support. Financial support from the European Union's Seventh Framework Program for research, technological development and demonstration under Grant Agreement No. ENER/FP7/284533/NEXT-BUILDINGS as well as from BMBF under Grant 13N14517 NANOSCALE is gratefully acknowledged.

Funding Open Access funding enabled and organized by Projekt DEAL.

Open Access This article is licensed under a Creative Commons Attribution 4.0 International License, which permits use, sharing, adaptation, distribution and reproduction in any medium or format, as long as you give appropriate credit to the original author(s) and the source, provide a link to the Creative Commons licence, and indicate if changes were made. The images or other third party material in this article are included in the article's Creative Commons licence, unless indicated otherwise in a credit line to the material. If material is not included in the article's Creative Commons licence and your intended use is not permitted by statutory regulation or exceeds the permitted use, you will need to obtain permission directly from the copyright holder. To view a copy of this licence, visit http://creativecommons.org/licenses/by/4.0/.

\section{References}

Amirzada, M.R., Tatzel, A., Viereck, V., Hillmer, H.: Surface roughness analysis of $\mathrm{SiO}_{2}$ for PECVD, PVD and IBD on different substrates. Appl. Nanosci. 6, 215-222 (2016). https://doi.org/10.1007/ s13204-015-0432-8 
Cheng, P., Yan, C., Wu, Y., et al.: Efficient and stable organic solar cells: via a sequential process. J. Mater. Chem. C 4, 8086-8093 (2016). https://doi.org/10.1039/c6tc02338j

Daniel, A.F., Fair Haven, N.J.: Solar Cell Array. US patent 2962539A, dated 29 Nov 1960

Gan, K.J., Liu, P.T., Chien, T.C., et al.: Highly durable and flexible gallium-based oxide conductive-bridging random access memory. Sci. Rep. 9, 1-7 (2019). https://doi.org/10.1038/s41598-019-50816-7

Ge, C., Cretu, E.: A sacrificial-layer-free fabrication technology for MEMS transducer on flexible substrate. Sens. Actuators A Phys. 286, 202-210 (2019). https://doi.org/10.1016/j.sna.2018.12.049

Hillmer, H., Al-Qargholi, B., Khan, M.M., et al.: Optical MEMS-based micromirror arrays for active light steering in smart windows. Jpn. J. Appl. Phys. 57, 1-13 (2018). https://doi.org/10.7567/JJAP.57. 08PA07

Iskhandar, M.S.Q., Al-qargholi, B., Khan, M.M., et al.: Development of Optical MEMS-Based Micromirror Arrays for Smart Window Applications: Implementation of Subfield Addressing and Reliability Measurement. Jahrb Oberflächentechnik, Leuze Verlag (yearb Surf Technol Leuze Publ house) (2019); ISBN: 978-3-87480-357-1

Koo, M., Il, P.K., Lee, S.H., et al.: Bendable inorganic thin-film battery for fully flexible electronic systems. Nano Lett. 12, 4810-4816 (2012). https://doi.org/10.1021/n1302254v

Liu, Y., Wang, H., Zhao, W., et al.: Flexible, stretchable sensors for wearable health monitoring: sensing mechanisms, materials, fabrication strategies and features. Sensors (2018). https://doi.org/10.3390/ s18020645

Lu, N., Kim, D.: Glenn omandam inventions, patents and patent applications-justia patents search. Sensors 1, 53-62 (2013). https://doi.org/10.1089/soro.2013.0005

Malik, A., Kandasubramanian, B.: Flexible polymeric substrates for electronic applications. Polym Rev 58, 630-667 (2018). https://doi.org/10.1080/15583724.2018.1473424

Park, J.H., Lee, H.E., Jeong, C.K., et al.: Self-powered flexible electronics beyond thermal limits. Nano Energy 56, 531-546 (2019). https://doi.org/10.1016/j.nanoen.2018.11.077

Shih, C.W., Chin, A.: Remarkably high mobility thin-film transistor on flexible substrate by novel passivation material. Sci. Rep. 7, 1-7 (2017). https://doi.org/10.1038/s41598-017-01231-3

Wang, X., Liu, Z., Zhang, T.: Flexible sensing electronics for wearable/attachable health monitoring. Small 13, 1-19 (2017). https://doi.org/10.1002/smll.201602790

Wojtyra, M.N., Lesyk, R.B.: Effects of deposition method of PECVD silicon nitride as MIM capacitor dielectric for GaAs HBT technology. Ecol Trans. 35, 229-240 (2017). https://doi.org/10.11603/23120967.2017.1.7533

Wong, W.S., Salleo, A.: Flexible Electronics: Materials and Applications, vol: 11. Springer, Boston (2009)

Yang, K., Kim, S., Kim, S.-Y., et al.: Flexible $\mathrm{Cu}_{2} \mathrm{ZnSn}(\mathrm{S}, \mathrm{Se})_{4}$ solar cells with over $10 \%$ efficiency and methods of enlarging the cell area. Nat. Commun. 10, 1-10 (2019). https://doi.org/10.1038/ s41467-019-10890-X

Yao, Y., Taylor, J., Cirullo, L., et al.: Ultra-thin, ultra-lightweight, and multifunctional skin for highly deformable structures. AIAA Scitech. (2019). https://doi.org/10.2514/6.2019-2028

Yaojian, L., Kang, C.: Semiconductor Device and Method of Forming Thick Encapsulant for Stiffness with Recesses for Stress Relief in FO-WLCSP (2016)

Zhou, L., Mao, J., Ren, Y., et al.: Recent advances of flexible data storage devices based on organic nanoscaled materials. Small 14, 1-27 (2018). https://doi.org/10.1002/smll.201703126

Zhu, B., Wang, H., Leow, W.R., et al.: Silk fibroin for flexible electronic devices. Adv. Mater. 28, 42504265 (2016). https://doi.org/10.1002/adma.201504276

Publisher's Note Springer Nature remains neutral with regard to jurisdictional claims in published maps and institutional affiliations. 